\title{
MEASUREMENTS OF THE EFFECT OF BOUNDARY CONDITIONS ON UPSTREAM AND DOWNSTREAM NOISE ARISING FROM ENTROPY SPOTS
}

\author{
Francesca De Domenico*, Erwan O. Rolland, Simone Hochgreb \\ Department of Engineering \\ University of Cambridge \\ Cambridge, CB2 1PZ \\ United Kingdom
}

\begin{abstract}
Pressure fluctuations in combustors arise either directly from the heat release rate perturbations of the flame (direct noise), or indirectly from the acceleration of entropy, vorticity or compositional perturbations through nozzles or turbine guide vanes (indirect noise). In this work, the generation of synthetic entropy spots via the Joule effect produces direct noise, and their acceleration through orifice plates and nozzles produces indirect noise. These acoustic waves reverberate, reflecting several times at the boundaries to add up to the measured pressure. Single travelling pulses are isolated by the introduction of a semiinfinite tube that acts as an anechoic termination for a limited time-window. It is shown how the shape of the converging nozzle does not affect the reflection of the direct noise wave, confirming the hypothesis of a compact nozzle. Further, it is demonstrated that the assumption of an isentropic nozzle does not hold, but that an alternative theory which takes into account the partial acoustic energy dissipation offers good agreement with the experiments. Finally, it is shown that the reflected indirect noise is underpredicted by isentropic theories. An extension of the present work is indicated for the measurement of the transmissivity of indirect noise.
\end{abstract}

\section{NOMENCLATURE}

$$
\begin{array}{ll}
\text { A } & \text { Area }\left[\mathrm{m}^{2}\right] \\
I & \text { Electric current }[\mathrm{A}]
\end{array}
$$

$L \quad$ Length [m]

$M \quad$ Mach number [-]

$P \quad$ Acoustic wave amplitude [-]

$\dot{Q} \quad$ Fluctuating heat release rate [W]

$R_{i} \quad$ Reflection coefficient of the $i^{t h}$ boundary [-]

Re Reynolds number [-]

$T$ Temperature [K]

$T_{i} \quad$ Transmission coefficient of the $i^{\text {th }}$ boundary [-]

$\mathrm{V}$ Voltage [V]

c Speed of sound $[\mathrm{m} / \mathrm{s}]$

$c_{p} \quad$ Specific heat $[\mathrm{J} / \mathrm{kg} / \mathrm{K}]$

$f \quad$ Frequency [1/s]

$\dot{m}$ Mass flow rate $[\mathrm{kg} / \mathrm{s}]$

$p$ Pressure $[\mathrm{Pa}]$

$\dot{q} \quad$ Normalised heat release [-]

$t_{a} \quad$ Anechoic time [s]

$t_{p} \quad$ Pulse length [s]

$u \quad$ Flow velocity $[\mathrm{m} / \mathrm{s}]$

$\gamma \quad$ Specific heat ratio $[\mathrm{J} / \mathrm{kgK}]$

$\Lambda \quad$ Non dimensional wavelength [-]

$\lambda$ Wavelength [m]

$(\cdot)_{\text {air }}$ Property of the air flow

$(\cdot)_{w} \quad$ Property of the wires

(.) Mean component

$(\cdot)^{\prime} \quad$ Fluctuating component

$(.)^{+}$Downstream travelling acoustic wave [-]

$(\cdot)^{-} \quad$ Upstream travelling acoustic wave $[-]$ 


\section{INTRODUCTION}

Acoustic perturbations arising from the heat release in combustion devices are a topic of increasing interest, particularly as a source of potential instabilities in combustors. The introduction of lean premixed pre-vaporised combustors, which produce low $\mathrm{NO}_{x}$ emissions but burn more unsteadily, has increased noise emissions and the potential for catastrophic instabilities. Pressure fluctuations in combustion chambers are classified as direct and indirect noise. The former arises directly from the heat release rate perturbations in the flame [1]. The latter is generated indirectly from the acceleration of regions of non-uniform temperature [2], density, composition [3] or vorticity [4] through a narrow passage such as turbine nozzle guide vanes. These sound waves are both transmitted downstream of the acceleration point, contributing to the overall noise emission [5], and reflected upstream, where they may couple with the acoustics of the system [6-8]. Marble and Candel [9] originally developed a one dimensional isentropic analytical model, deriving expressions for the magnitude of both direct and indirect noise in the low frequency limit, and more recent work has revisited the issue $[6,8,10,11]$. One of the main obstacles in the investigation of entropy noise is the lack of experimental data due to the complex dynamics of combustion chambers and the consequent difficulties in the separation between direct and indirect noise in the acquired pressure traces.

To overcome this issue, simplified laboratory scale experiments have been designed to separate the contributions of direct and indirect noise, and to test existing analytical and numerical models [12-17]. This paper refers to the configuration described in [16], which demonstrate that a pulsating heating grid in a flow generates acoustic waves (direct noise) and temperature nonuniformities (hot spots) which produce sound when accelerated through an orifice plate (indirect noise). The convective time of the entropy spots for travelling from their origin to the contraction is designed to be much longer than the heating pulse interval, to ensure a clear time separation between the contribution of direct and indirect noise in the acquired pressure signal. The resulting signal is the integral of the original pulse combined with the multiple acoustic reflections (reverberation) of the direct and indirect noise arising at the boundaries of the system (Fig. 2) [17]. The term direct noise is used here to indicate the pressure response due to the sound waves generated by the change in heat release at the heating grid, including the original acoustic pulses overlapped with the corresponding reflected waves. Similarly, the term indirect noise indicates the pressure perturbation due to the acceleration of the hot spots: the original travelling waves combined with the corresponding reflected waves at the boundaries. Understanding the pressure signal therefore requires good characterisation of the fate of the corresponding oscillations at the boundary.

The long cooling times of the heated wires require operation of the pulsed heating at unusually low frequencies in order to produce controlled and measurable temperature (thus pressure) pulses. Yet reflection coefficients at boundaries are not well characterised in this regime as conventional multiple-microphone methods are impractical for long wavelengths. In order to overcome this problem, a 30-meter-long tube termination is attached to the downstream end of the test tube to produce an effectively anechoic end over a limited time interval. The roundtrip time of the resulting sound waves to the termination of the 30-metre-long tube is $0.18 \mathrm{~s}$. This allows sufficient time for the downstream and upstream running waves to be well separated, obtaining clean measurements of the corresponding pressure histories for system identification and modelling.

The pressure histories obtained with the time-based anechoic termination are presented in the first part of the paper. The second part of the work investigates the effect of the different shapes of acceleration profiles: converging nozzles and orifice plates with the same inlet and throat areas, as well as a convergent/divergent nozzle. The latter enables the acquisition of reflected and transmitted pressure traces and the comparison with existing models.

\section{EXPERIMENTAL SET-UP}

The experimental set-up, presented in [16], is shown in Figure 1. Air flows through a tube at a controlled rate and passes through a heating device. When current is pulsed into this heating module it generates a heat release pulse and a hot spot via the Joule effect, which is then advected with the mean flow. If this hot spot is accelerated through a nozzle, it produces indirect noise. Dynamic pressure measurements are performed downstream of the heating device, via flush-mounted pressure transducers, at locations indicated in figure 1.

Filtered compressed air from the laboratory air supply system is fed into a $250 \mathrm{~L}$ tank to dampen out upstream pressure oscillations. The tank pressure is set to 5 bar using a pressure gauge. The tank is connected via a $12 \mathrm{~mm}$ inner diameter plastic hose to a mass flow controller (MFC, Alicat MCR500, accuracy, $\pm 1 \%$ full scale). The MFC is connected to the test section via a $12 \mathrm{~mm}, 1.2$ meter long plastic hose with a flat flange and provide an acoustic boundary condition with a reflection coefficient near unity $[16,17]$. The tube has an inner diameter of $42.6 \mathrm{~mm}$ and is made from sections of PVC and stainless steel 316. The heating device is fitted $700 \mathrm{~mm}$ downstream of the tube inlet in a PVC flange for electric isolation. The device itself is composed of three grids of thin tungsten wires ( $58 \mu \mathrm{m}$ diameter) connected in series, with an overall resistance of about $1 \Omega$. Each grid is made of $2.3 \mathrm{~m}$ of wire wound around a FR4 substrate $1.6 \mathrm{~mm}$ thick, which keeps the wires in place using a toothed comb structure. A schematic of a single heating grid is shown in figure 1. An in-house circuit controlled by a computer drives a power supply (Glassman Lp 60-20), and delivers a current pulse of 21 A to the heating device. The pulse length is chosen to be $t_{p}=0.1 \mathrm{~s}$. 

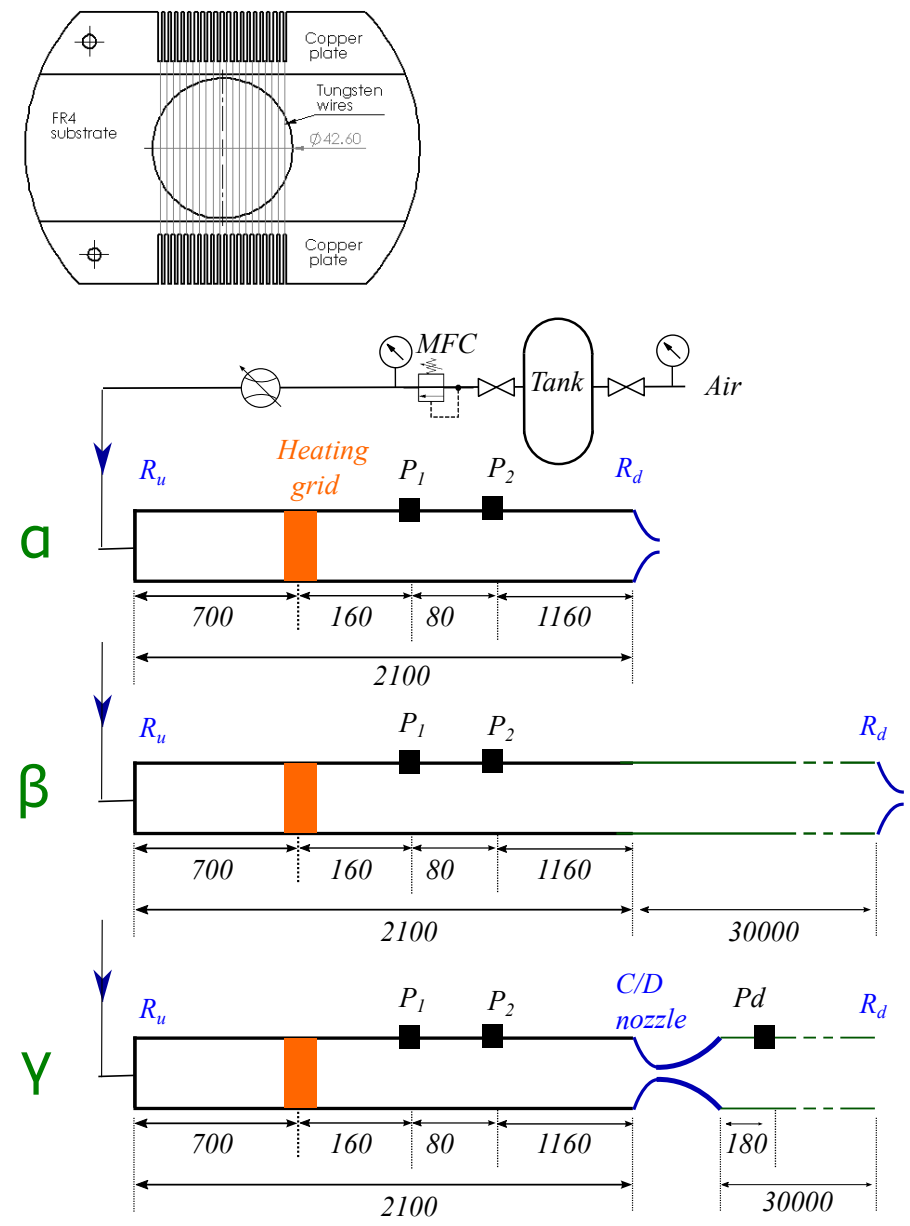

FIGURE 1. SCHEMATIC OF THE EXPERIMENTAL CONFIGURATIONS ANALYSED $(\alpha, \beta, \gamma)$. ZOOM ON THE HEATING GRID MODULE. DIMENSIONS IN MM. NOT TO SCALE.

The pressure signal is acquired with piezoresistive pressure transducers that have been shown to be appropriate for the low frequency pulses [16]: three Kulite XTE-190(M) piezoresistive pressure transducers (0-3.5 bar), (mounted at locations $P_{1}, P_{2}$ and $P_{d}$ in figure 1$)$ and a Kulite XT-140M piezoresistive absolute pressure transducer ( $0-10$ bar) (mounted at location $\left.P_{1}\right)$. The outputs of the piezoresistive pressure transducers are amplified with a Fylde FE-379-TA modular DC amplifier, and transferred using a NI PCI-5259 board via a NI-2090 DAQ box at 16-bit resolution and 8192 samples per second. As piezoresistive pressure transducers have limited sensitivity, the pressure signals are averaged over 80 pulses and filtered with a $0-100 \mathrm{~Hz}$ rectangular window digital filter.

Three configurations are considered, labeled $\alpha, \beta$ and $\gamma$, with different terminations and lengths, as shown in figure 1 and described below.

\subsection{Basic set-up (Configuration $\alpha$ )}

For configuration $\alpha$, different end terminations at the end of the rig, located $1.4 \mathrm{~m}$ from the heater, are tested. They are all characterised by the same throat area of $6.6 \mathrm{~mm}$ diameter: 5 convergent nozzles with different contraction shapes and lengths, a convergent/divergent nozzle and two orifice plates (thick and thin). The different termination shapes are described in Appendix A.

\subsection{Anechoic end terminated set-up (Configuration $\beta$ )}

In a recent paper [17], we have shown that it is necessary to know the reflection coefficients of the boundaries in order to compare the acquired direct and indirect noise with theoretical predictions in a reverberating environment. By increasing the distance between these two boundaries, the time separation between the original wave and its reflection at the downstream boundary becomes sufficiently large, so that each pulse can be easily identified and time separated from the others.

A 30 meter long tube is used to achieve this time separation, in the form of a $45 \mathrm{~mm}$ inner diameter PVC tube (RS lightweight delivery hose reinforced with a rigid internal crush and kink resistant white PVC helix). As a first approximation, it is assumed that the difference in the cross section between the PVC tube (42.6 $\mathrm{mm}$ diameter) and the long green hose $(45 \mathrm{~mm}$ diameter) can be neglected. The hose is coiled with a radius of 0.6 $\mathrm{m}$ : there is no evidence in the literature suggesting that such a large bend radius might significantly affect the propagation of the sound waves. Over such a long length, the hot spots are entirely dissipated before the far end of the tube, eliminating the contribution of the indirect noise. Moreover, since the frequency spectrum of the signal is mainly in the $0-50 \mathrm{~Hz}$ frequency range, the corresponding wavelengths are at least one order of magnitude longer than this pipe, so the shapes of the reflected waves are not substantially distorted. The working principle of this end termination is similar to the semi-infinite tube of probe microphones $[18,19]$ but, since the diameter is large and the frequency low, the acoustic energy is not sufficiently dissipated, and it is possible to observe reflections arising at the termination of the tube.

The anechoic time $t_{a}$, defined as the time required for a sound wave to reach the end of the long tube and be reflected back, is approximately

$$
t_{a}=\frac{L_{t o t}}{c} \simeq \frac{2 \times(30+1.4)}{343} \simeq 0.183 \mathrm{~s}
$$

The heating pulse length $t_{p}=0.1 s$ is chosen to be shorter than $t_{a}$, so the downstream running acoustic waves generated at the heating grid are not superposed with the corresponding reflected 


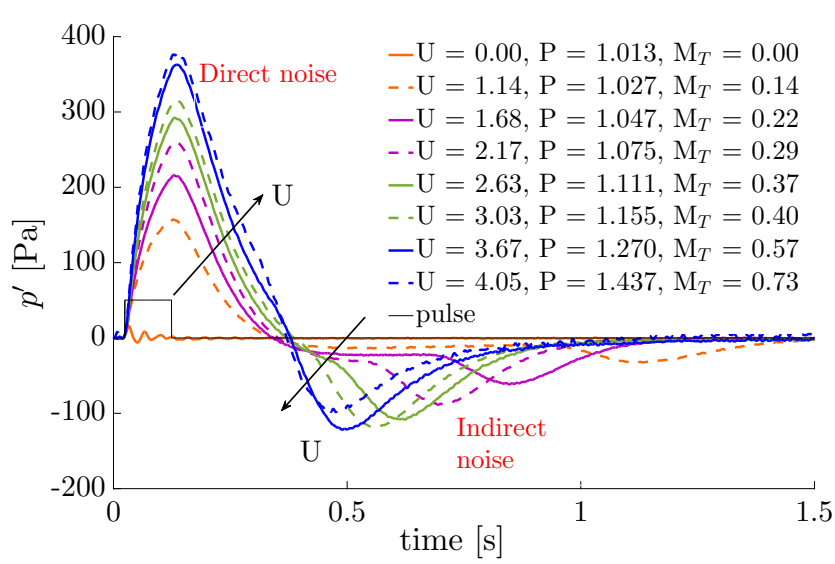

FIGURE 2. DIRECT AND INDIRECT NOISE MEASURED UPSTREAM OF THE THICK ORIFICE PLATE TERMINATION AFTER PULSES OF $0.1 \mathrm{~s}$. CONFIGURATION $\alpha$. LEGEND: VELOCITY [M/S], PRESSURE [BAR] AND THROAT MACH NUMBER [-] [16]

ones. This allows further insight on the behaviour of the system: a real anechoic termination is ideal for the characterisation of acoustic sources, but it is not possible to build a totally nonreflective boundary condition for the wavelengths characteristic of this experiment [20]. Using this long tube termination, the downstream end is silent (behaves as anechoic) for $t<t_{a}$ allowing the isolation of single travelling waves.

\subsection{Convergent/divergent nozzle set-up (Configuration $\gamma$ )}

The transfer function of a convergent/divergent nozzle has been investigated in several analyses [9, 21], both for the direct and the indirect noise generated. A convergent/divergent nozzle is therefore attached at the end of the $1.4 \mathrm{~m}$ long tube, enabling the acquisition of both the reflected $\left(p_{u}\right)$ and the transmitted $\left(p_{d}\right)$ pressure trace for comparison with models.

\section{RESULTS: REVERBERATION OF WAVES}

\subsection{Previous results: configuration $\alpha$ [16]}

In a previous paper, direct and indirect noise were obtained in configuration $\alpha$, terminated with a thick orifice plate (O2), after a heating pulse interval of $0.2 \mathrm{~s}$ [16]. The acquired pressure traces correspond to the sum of multiple waves generated by direct and indirect noise and their reverberation at the orifice and upstream boundary. The round trip time $t_{\text {tot }}$ of a sound wave in the $\alpha$ set-up is $t_{\text {tot }}=2 \cdot L_{t o t} / c \simeq 0.012 \mathrm{~s}$, which is one order of magnitude smaller than the pulse length and the convective time: the acoustic pulses therefore reverberate several times at the boundaries and the pressure signal builds up during that interval. [17].

Figure 2 shows the dynamic pressure obtained upstream of the orifice, following a $t_{p}=0.1 \mathrm{~s}$ heating pulse for a fixed power input into the heating device $(\mathrm{V}=35 \mathrm{~V}, \mathrm{I}=21 \mathrm{~A})$. The flow is in subsonic conditions. Direct (positive) and indirect (negative) noise can be clearly identified in the measured pressure trace, since the convective time is chosen to be longer than the pulse length.

In configuration $\alpha$, the direct noise signal arising from the activation of the heating device always peaks at the end of the heating pulse. The indirect noise signal generated from the acceleration of the hot spots through the nozzle peaks at point coinciding with the convective time for the hot spots to travel from the heating grid to the orifice plate. Therefore, an increase in velocity leads to a shift in the indirect noise peak to earlier times. In order to clearly analyse the behaviour of individual pulses, without the effect of multiple reflections, configuration $\beta$ is considered in the next section.

\subsection{Effect of multiple reflections on the shape of the signal}

The rapid heating of the grid of wires generates upstream and downstream running compression waves which travel with the speed of sound relative to the air flow, and are reflected and transmitted when they encounter a boundary. The reverberation of individual heating-induced compression waves has already been observed in confined environments with static gas (denoted as thermoacoustic convection [22-24]). The mean flow enhances the heat transfer between the heated surface and the gas, so the generated compression waves are of higher intensity than in a static gas. The upstream $P^{-}$and downstream $P^{+}$running acoustic waves are defined as [11]

$P^{+}=\frac{1}{2}\left(\frac{p^{\prime}}{\gamma \bar{p}}+\frac{u^{\prime}}{\bar{c}}\right)=\frac{1}{2} \frac{\bar{M}}{1+\bar{M}} q^{\prime} ; \quad P^{-}=\frac{1}{2}\left(\frac{p^{\prime}}{\gamma \bar{p}}-\frac{u^{\prime}}{\bar{c}}\right)=\frac{1}{2} \frac{\bar{M}}{1-\bar{M}} q^{\prime}$

where $q^{\prime}=\frac{Q^{\prime}}{\dot{m} c_{p} T_{0}}$ is the normalised heat release rate, $Q^{\prime}$ the heat release rate perturbation, $\dot{m}$ the mass flow rate, $c_{p}$ the specific heat at constant pressure, and $T_{0}$ the initial temperature. Figure 3 illustrates this behaviour, obtained with configuration $\beta$. $P^{+}$and $P^{-}$appear as rectangular waves that recall the heating pulse, but reflections modify the overall shape and amplitude of the pressure trace. The upstream traveling wave $P^{-}$ reaches the upstream boundary, which has a reflection coefficient close to unity $\left(R_{u} \simeq+1\right)$, and is positively reflected back within $t_{u}=2 L_{u} / c \simeq 0.004 \mathrm{~s}$. Since this travel time is much shorter than the heating interval, the two waves $R_{u} P^{-}$and $P^{+}$move downstream as one. The passage of the positively added waves is measured by the pressure transducer (acoustic pulse) as they travel to the downstream end (figure 3 ). When the 30 meter long tube is left open, these two waves $\left(R_{u} P^{-}\right.$and $\left.P^{+}\right)$are negatively reflected back $\left(R_{d} R_{u} P^{-}\right.$and $\left.R_{d} P^{+}\right)$with nearly the same amplitude $\left(R_{d} \simeq-1\right)$, since the open end constitutes a node for the pressure oscillations. $R_{d} R_{u} P^{-}$and $R_{d} P^{+}$pass again over the same trans- 


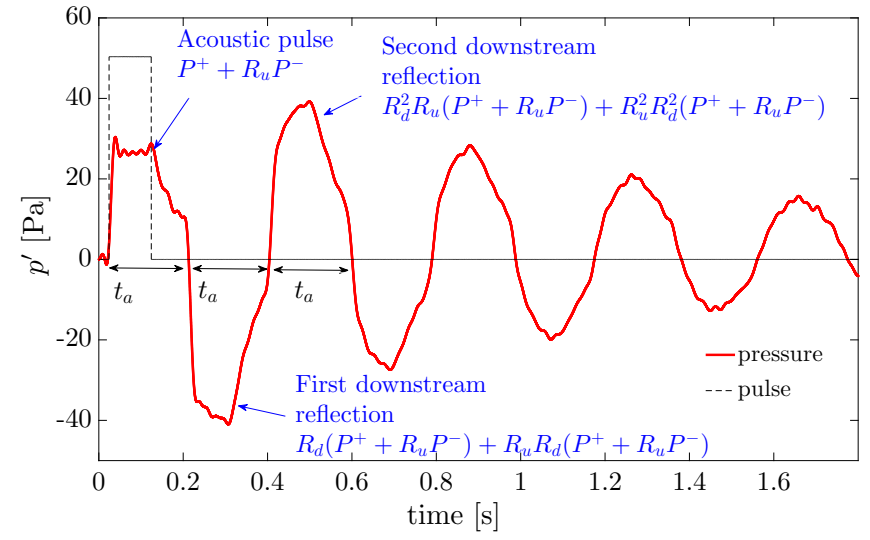

FIGURE 3. INTERFERENCE OF SOUND WAVES DUE MULTIPLE REFLECTIONS. CONFIGURATION $\beta$, OPEN END TERMINATION. $\mathrm{U}=1.64 \mathrm{M} / \mathrm{S} . t_{p}=0.1 \mathrm{~s}$

ducer after $t \simeq t_{a}$. However, since the travel time between the transducer and the upstream boundary is very short relatively to the travelling pulse length (after $\left.t_{u}\right)$, they are again nearly immediately reflected at the upstream boundary and detected at that location. Therefore, the amplitude of the second oscillation is higher than the amplitude of the first oscillation despite the fact that the heating device is now turned off and no further energy is delivered to the system: four waves pass simultaneously over the pressure transducer $\left(R_{d} R_{u} P^{-}, R_{d} P^{+}, R_{d} R_{u}^{2} P^{-}\right.$and $\left.R_{d} R_{u} P^{+}\right)$. These waves continue to repeatedly reflect at the boundaries, until viscous and thermal losses dissipate their acoustic energy entirely. This behaviour can be observed in figure 3 , where the system is excited by a square pulse of interval $t_{p}=0.1 \mathrm{~s}$, which is shorter than the anechoic time $t_{a}$, so that the heating device is switched off before the reflected negative wave reaches the measurement point. The first positive oscillation has a square shape that resembles the heating pulse, and the pressure rises sharply when the heating device is activated. When the heating grid is switched off, the pressure starts decaying smoothly until, at $t=t_{a}=0.183 \mathrm{~s}$, the pressure decreases sharply upon the arrival of the first upstream propagating wave. The amplitude of this negative peak is higher in its absolute value than the first positive peak, as expected. The pattern repeats itself, with a lower amplitude owing to acoustic losses.

\subsection{Effect of the mass flow rate on the measured up- stream pressure signal}

An increase in the mass flow rate for a constant input power increases both the direct and the indirect noise in the reverberating system (Configuration $\alpha$, Fig. 2) and in the non-reverberating system (Configuration $\beta$, figure 4 and 5): the mean pressure in the duct (when terminated with a orifice or a nozzle), the reflec-

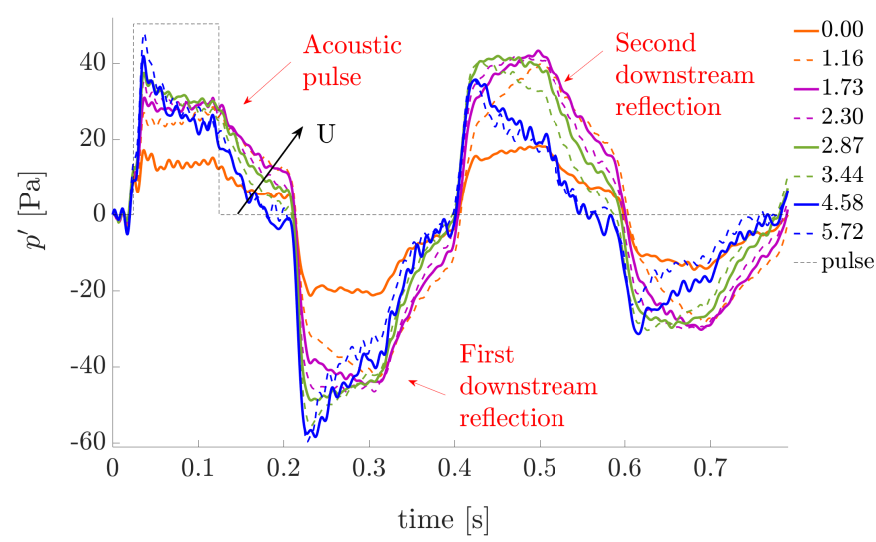

(a)

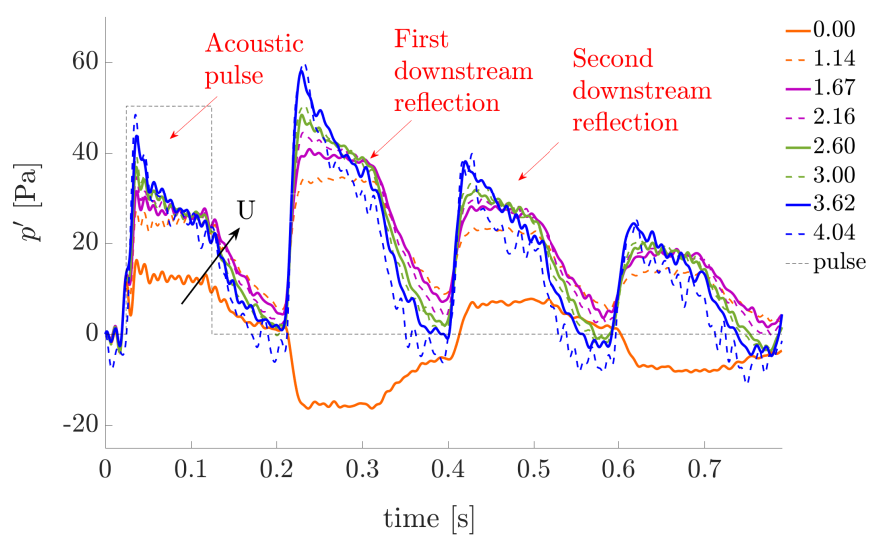

(b)

FIGURE 4. EFFECT OF THE MASS FLOW RATE ON THE PRESSURE TRACES ACQUIRED FOR A FIXED POWER. CONFIGURATION $\beta$ WITH OPEN END TERMINATION (a) AND THICK ORIFICE END TERMINATION (b). LEGEND: FLOW VELOCITY [M/S]

tion coefficient of the orifice plate [25] and the convective heat transferred to the air flow all increase, while the cooling time of the heating grid, the peak temperature of the hot spots and their travelling time decrease. In the reverberating system (configuration $\alpha$, figure 2), it is difficult to assess which of these effects affects the generated direct and indirect noise, since multiple waves are overlapped.

Configuration $\beta$ allows a separation of the various effects (figure 4). The 30-meter-long duct is terminated with two different ends: an open end (figure 4(a)), and the thick orifice plate $\mathrm{O} 2$ (figure 4(b)). While the acoustic pulses are negatively reflected back at the open end (figure 4(a)), they are positively reflected back at the orifice (figure 4(b)). This highlights the effect of reverberation on the overall signal: the constructive interference of the multiply reflected acoustic pulses in the shorter tube (configuration $\alpha$ ) amplifies the signal nearly 10 -fold. The downstream- 


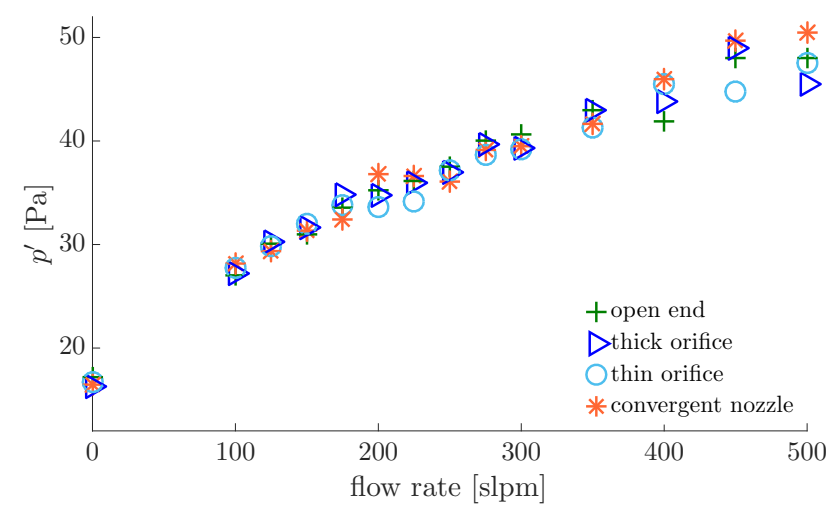

FIGURE 5. PRESSURE PEAK OF THE FIRST PULSE VS MASS FLOW RATE FOR FOUR DIFFERENT END TERMINATIONS. CONFIGURATION $\beta$.

running travelling wave $R_{u} P^{-}+P^{+}$is isolated (Acoustic pulse in figure $4(\mathrm{a}-\mathrm{b}))$. The combined acoustic wave $R_{u} P^{-}+P^{+}$always peaks in the first few milliseconds of the pulse, and its peak increases with mass flow rate (figure 5). Taking the upstream reflection coefficient as $R_{u} \simeq(1-\bar{M}) /(1+\bar{M}) \simeq+1$ [17], the downstream travelling wave acquired from the pressure transducer can be approximated as $P^{\prime}=R_{u} P^{-}+P^{+} \simeq P^{-}+P^{+}$. From Eq. 2, when $\bar{M}<<1$ the peak in the first oscillation in the pressure trace is

$$
p^{\prime}=\frac{\bar{M}}{1-\bar{M}^{2}} \frac{Q^{\prime}}{\dot{m} c_{p} T} \gamma \bar{p} \simeq \frac{\gamma-1}{c} \frac{Q^{\prime}}{A}
$$

where $Q^{\prime}$ is the heating power transferred from the heating grid to the air, and $A$ the cross sectional area. Equation 3 shows that the unsteady heat release of the heating grid generates direct noise also in the absence of mean flow (in this case, $Q^{\prime}$ is the heating rate between the grid and the air trapped in the duct via natural convection). The presence of mean flow in the duct increases the heat power transferred from the heating grid to the flow: using a lumped-parameter analysis [26]

$$
Q^{\prime}=h(R e) A_{w}\left(T_{w}-T_{\infty}\right)
$$

where $h$ is the heat transfer coefficient, which increases with the Reynolds number $R e, A_{w}$ is the exchange area, $T_{w}$ is the wire temperature and $T_{\infty}$ is the air flow temperature before encountering the heating grid. Figure 5 shows the amplitude of the first pressure peak for four end terminations (open end, thick orifice, thin orifice and convergent nozzle termination). The duct is at atmospheric conditions with an open end, but is pressurised

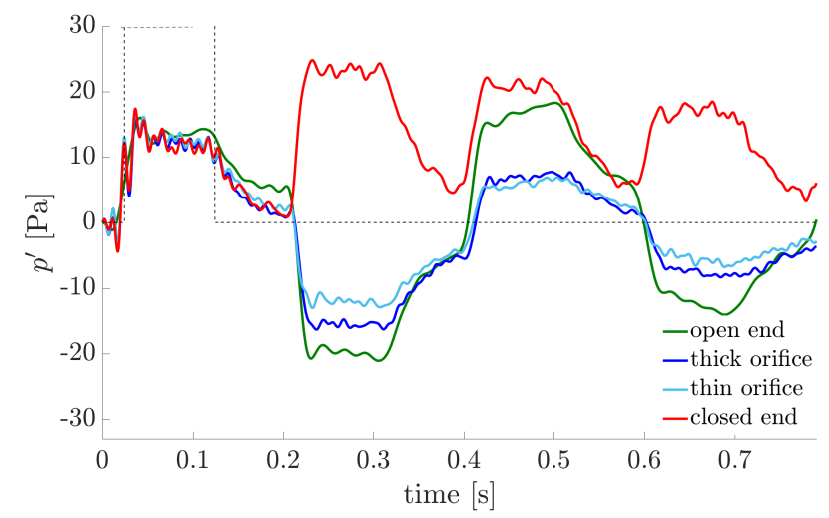

FIGURE 6. PRESSURE HISTORIES FOR THE ZERO MEAN FLOW CASE FOR FOUR DIFFERENT DOWNSTREAM END TERMINATIONS. CONFIGURATION $\beta$.

when terminated by orifices and nozzles. However, the magnitude of the pressure peaks is only a function of the mass flow rate: both in the pressurised (nozzle-orifice terminated) and in the non-pressurised (open exit) cases, the oscillations have the same amplitude for a given mass flow rate. The lack of dependence on pressure is related to the fact that the typical rise in pressure expected by the steady momentum equation needs to be communicated to the flow via the reflected pressure pulse which in this case has not yet arrived.

\subsection{Zero mean flow case}

Figure 4(b) shows that the acoustic pulses are positively reflected back on the orifice plate in the case of non-zero mean flows and reflected back as negative waves in the case with zero mean flow, as if they had encountered an open boundary.

To explain this behaviour, the pulsed heating-experiment is repeated without mean flow for four different end terminations (in configuration $\beta$ ): open end, closed end, thin and thick orifice (figure 6). The open and closed ends are respectively a node and antinode for the pressure oscillations: acoustic waves are negatively reflected back at the open end $\left(R_{d} \simeq-1\right)$ and positively mirrored back at the closed end $\left(R_{d} \simeq+1\right)$. In the case of orifice plates, the reflected pulses have an opposite sign with respect to the incident pulse, as in the case of the open end termination case, but with a smaller amplitude, which suggests that the amount of acoustic energy reflected back is lower. Salikuddin et al. $[27,28]$ showed that, when a sound wave encounters an orifice plate without mean flow, the portion of the wave that impacts on the closed wall is mirrored back $\left(R_{d} \simeq+1\right)$, while the remaining encounters the open hole and is reflected back with negative sign $\left(R_{d} \simeq-1\right)$. The total reflected wave is the resultant of these inand out-of-phase waves, which causes some amount of low fre- 
quency acoustic energy cancellation. Moreover, if the acoustic energy is high enough, part of it is dissipated by the conversion into vortical energy at the edges, where the flow separates. Leung et al. [29] demonstrated that, in the zero mean flow case, vortices are generated by an impinging acoustic pulse both upstream and downstream of the orifice, whereas with a mean flow, these vortices orientate themselves in the flow direction.

The zero mean flow behaviour observed in the experiments [27] can be predicted with the additions of a nonlinear term [30] to linear models for orifice plate reflections [31-33]. With mean flow, this nonlinear effect disappears. These mechanisms are particularly relevant for the current experiment, since vortices are stronger at low frequencies and for small apertures [29, 34]. In particular, the absorption of the kinetic energy in the form of turbulence at low frequencies constitutes a considerable sink of energy, which cannot be neglected.

Considering a vena contracta factor $\Gamma=0.8$ for the thick orifice and $\Gamma=0.65$ for the thin orifice $[25,35]$, the non-linear model for the acoustic impedance of an orifice without mean flow [30] predicts a reflection coefficient $R_{O 2} \simeq-0.62$ for the thick orifice and $R_{O 1} \simeq-0.5$ for the thin orifice. Although the long tube cannot be used to obtain absolute information on the reflection coefficients due to the inevitable dissipation of the acoustic energy occurring throughout its length, semi-quantitative information can be obtained from a relative comparison with the reflected peak amplitude $p_{r}^{\prime}$ due to an incident pulse $p_{i}^{\prime}$ for the open end case. As for an open end $R_{o e} \simeq-1$, the reflection coefficient $R_{x}$ of the $x^{\text {th }}$ termination can be approximated as:

$$
\frac{R_{x}}{R_{o e}}=\frac{\left(\frac{\left|p_{r}^{\prime}\right|}{\gamma p_{i}^{\prime}}\right)_{x}}{\left(\frac{\left|p_{r}^{\prime}\right|}{\gamma p_{i}^{\prime}}\right)_{o e}} \simeq \frac{\left|p_{r}^{\prime}\right|_{x}}{\left|p_{r}^{\prime}\right|_{o e}}
$$

Using Eq. 5, a reflection coefficient $R_{O 2} \simeq-0.67$ is obtained for the thick orifice, $R_{O 1} \simeq-0.57$ for the thin orifice and $R \simeq+0.92$ for the close wall. These values, despite being approximate, are perfectly consistent with the predictions.

\section{RESULTS: BOUNDARY CONDITIONS}

The previous sections emphasised how multiple reflections can significantly affect both the shape and amplitude of the acquired pressure signal. The knowledge of reflection coefficients appears to be crucial to understand and model the system. However, traditional techniques such as the two and multimicrophone methods fail in the ultra low frequency range of the experiment, as they require a spatial separation of the probes of at least $\lambda / 4[36,37]$, which is not feasible for the wavelengths of the experiment. As discussed in Sec. 2.4, the reflective properties of the boundary conditions obtained at high frequencies cannot be extended to the low frequency range because non linear phenomena substantially alter the reflective properties. At the current state of the art there is no reliable way to experimentally determine these reflection coefficients accurately enough in the low frequency range, so they need to be deduced a posteriori from the acquired pressure data using existing models for validation $[9,11,25,32]$. In the frequency range of the experiment, these models traditionally assume the validity of the compactness hypothesis, both for the direct and the indirect noise generated. The compactness hypothesis requires the nozzle longitudinal extension to be at least one order of magnitude smaller than the characteristic wavelengths of the experiment. In the current setup, taking $L_{n}=0.05 \mathrm{~m}$ as nozzle length, $f=1 / t_{p}=10 \mathrm{~Hz}$ as characteristic frequency and $U=1 \mathrm{~m} / \mathrm{s}$ as characteristic velocity, the non-dimensional characteristic wavelengths for direct noise $\left(\Lambda_{d}\right)$ and indirect noise $\left(\Lambda_{i}\right)$ become:

$$
\Lambda_{d}=\frac{c / f}{L_{n}} \simeq 700 \quad \Lambda_{i}=\frac{u / f}{L_{n}} \simeq 2
$$

The acoustic wavelength $\lambda_{d}=c / f$ is two orders of magnitude larger than the nozzle length, so the nozzle should be seen as a compact discontinuity from the point of view of the acoustic waves. However, this is not strictly valid for the indirect noise, because the convective wavelength $\lambda_{i}=u / f$ and the nozzle length are of similar magnitude. Physically, this means that the acceleration of the hot spots does not occur instantaneously, since the flow is too slow to see the nozzle as a compact jump. This suggests that the shape of the nozzle, and therefore the acceleration profile of the hot spots may influence the shape and amplitude of the indirect noise generated, as shown in previous numerical studies [38,39].

Two experiments are carried out to investigate the effect of boundary conditions on the acquired direct and indirect noise. In the first one, the duct (configuration $\alpha$ ) is terminated with the seven different converging shapes described in Appendix A (five convergent nozzles, N1, N2, N3, N4, N5 and two orifice plates, $O 1$ and $O 2$ ), while in the second one the duct (configuration $\gamma$ ) is terminated with the convergent/divergent nozzle, with (CD N1) and without (CD N2) the long tube termination at the end. Each test is performed over a range of 14 different mass flow rates. Figure 7 shows the pressure increase in the duct versus the mass flow rate for the different experiments. Although all these terminations have the same throat area, they have different vena contracta factors (or conversely, effective areas), as verified by the different pressure increases obtained for the same mass flow rate. In particular, the pressure increase obtained with the thin orifice plate in the duct is considerably higher than for the other cases: as already seen in Sec. 2.4 the vena contracta of a thin orifice is considerably smaller than unity [35]. The thick orifice, instead, behaves nearly as a convergent nozzle. The losses 


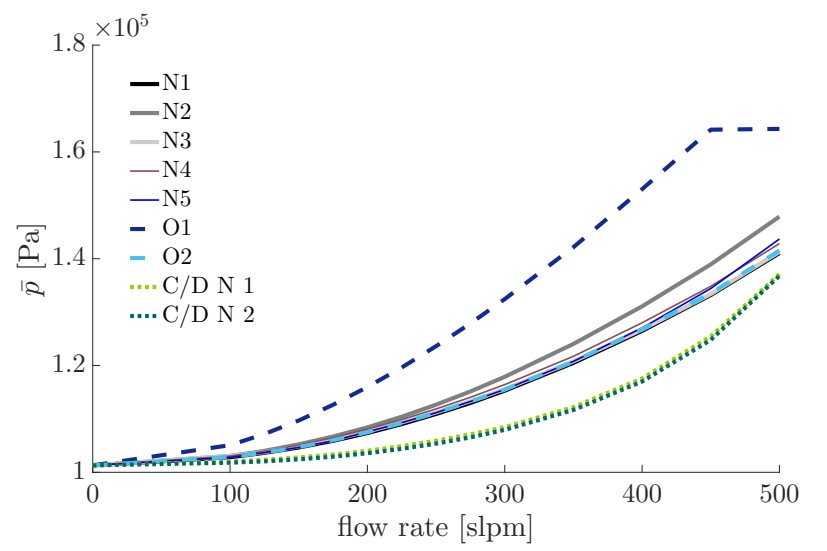

FIGURE 7. MEAN PRESSURE IN THE DUCT AS A FUNCTION OF MASS FLOW RATE FOR DIFFERENT END TERMINATIONS (N1-5, O1-2, CONFIGURATION $\alpha$; C/D N1, C/D N2 CONFIGURATION $\gamma$, WITH AND WITHOUT THE LONG TUBE TERMINATION RESPECTIVELY)

are less pronounced in the case of a convergent/divergent nozzle, explaining why the pressure increase in the duct is lower with the convergent/divergent nozzles. Figures $8(a-b)$ show an example of the pressure oscillations acquired for a constant mass flow rate (175 slpm and $400 \mathrm{slpm}$ respectively) for the different configurations. The maximum value of these traces corresponds to the direct noise peak (figure 9(a)). The minimum value of these traces, at the time corresponding to the convective time, is taken as representative of the indirect noise (figure 9b), even though this constitutes an approximation, since, for small convective times (high velocities), the negative oscillation of the indirect noise is partially overlapped with the direct noise. These are compared in the next subsection.

\subsection{Comparison of direct and indirect noise gener- ated with seven converging shapes}

In this section, the validity of the compactness hypothesis is investigated, comparing the shape and amplitudes of direct and indirect noise generated with the seven converging shapes (N1, N2, N3, N4, N5, O1 and O2). Figure 9(a) shows that all five convergent nozzles and the thick orifice plate behave nearly in the same way with respect to direct noise, while the values obtained with the thin orifice O1 (dashed line) is substantially higher, due to the smaller effective area (smaller vena contracta factor). In all other six cases $\mathrm{N}$ and $\mathrm{O}$, however, the direct noise amplitudes are comparable (figure 9). From these results it can be concluded that for the reflected direct noise the different end terminations behave as compact sources regardless of the contraction shape, provided that they have the same effective throat area. This confirms that the thick orifice plate used in [16] be-

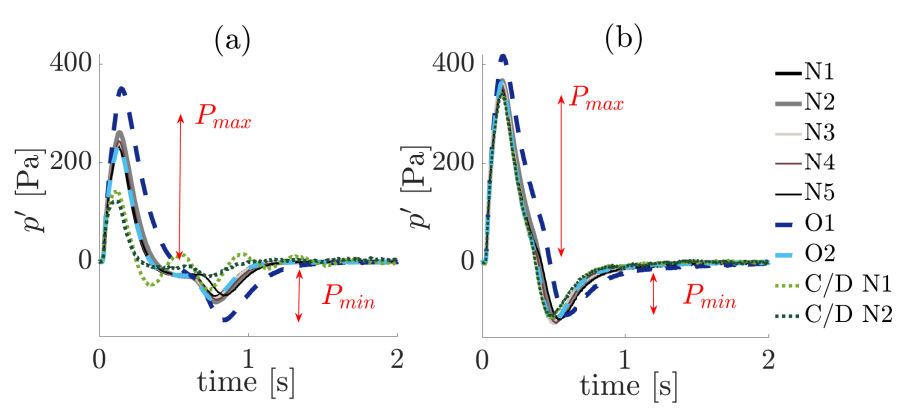

FIGURE 8. PRESSURE OSCILLATIONS ACQUIRED UPSTREAM OF THE NOZZLE WITH SEVEN DIFFERENT END TERMINATIONS FOR A MASS FLOW RATE OF 175 SLPM (a) AND 400 SLPM (b) (N1-5, O1-2, CONFIGURATION $\alpha$; C/D N1, C/D N2 CONFIGURATION $\gamma$, WITH AND WITHOUT THE LONG TUBE TERMINATION RESPECTIVELY)

haves acoustically as a convergent nozzle, as estimated from the compact theory [25,32]. Rolland [17] showed that the reflection coefficient of the thick orifice could be well predicted using the results of Bechert and Durrieu et al. [25,31].

Similar conclusions can be drawn regarding upstream travelling indirect noise 9(b): all $\mathrm{N}$ and $\mathrm{O}$ cases generate similar indirect noise, except for the thin orifice plate $\mathrm{O} 1$ (dashed line). This is a result of the higher reflection coefficient of the thinner orifice plate, as illustrated in (figure 8): the indirect noise waves reflected are more intense than in other cases.

Finally, we discuss the behaviour of the converging/diverging nozzle in configuration $\gamma$ with a long extension, which generates lower direct and indirect noise, as shown in Figs. 7-9.

\subsection{Reflected and transmitted direct and indirect noise with a convergent/divergent nozzle}

3.2.1 Direct noise contribution The acoustic behaviour of the system terminated with a convergent/divergent nozzle is analysed using Configuration $\gamma$ : one with (C/D N1) and without the 30 meter long termination (C/D N2) attached downstream of the divergent section. In these cases, an additional transducer is placed downstream of the nozzle ( $p_{d}$ in figure 1) in order to monitor the transmitted noise. In the absence of the long termination (C/D N2), the acoustic pulses transmitted through the nozzle are immediately reflected back at the downstream open end with opposite sign and same amplitude, effectively identical to configuration $\alpha$. In this configuration, the downstream transducer measures a positive and negative waves of the same amplitude and time separated of only $\tau=0.5 \mathrm{~ms}$, and these two oscillations effectively cancel out. The addition of the long termination allows the acoustic waves sufficient time to re- 


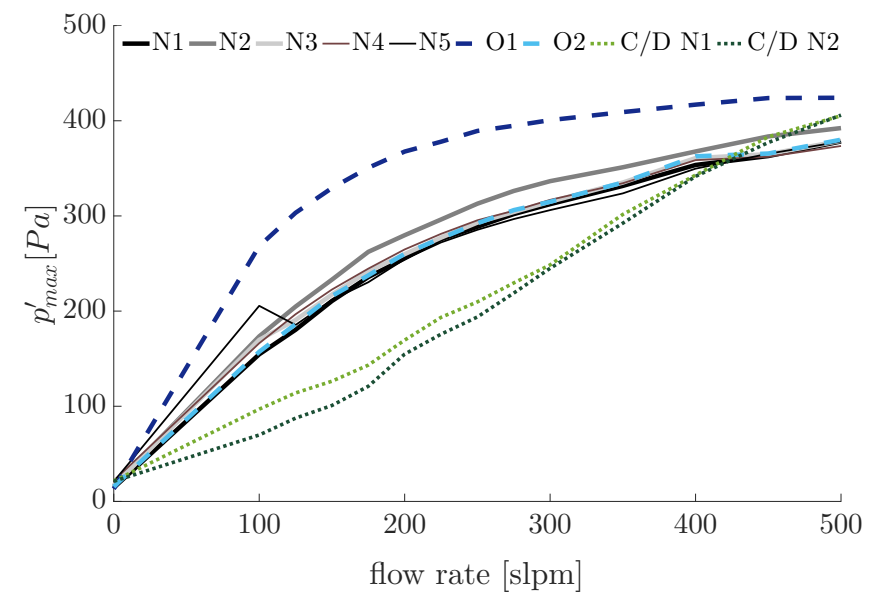

(a)

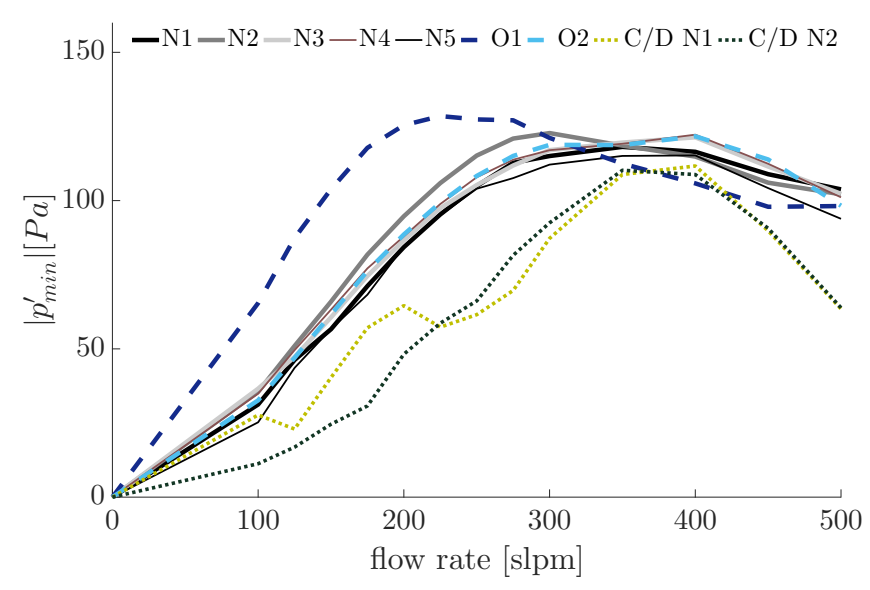

(b)

FIGURE 9. MEASURED PEAK PRESSURE FOR DIRECT (a) AND INDIRECT (b) NOISE OSCILLATIONS, FOR DIFFERENT END TERMINATIONS (N1-5, O1-2, CONFIGURATION $\alpha$; C/D N1, C/D N2 CONFIGURATION $\gamma$, WITH AND WITHOUT THE LONG TUBE TERMINATION RESPECTIVELY)

flect back from the open end ( $\tau \simeq t_{a} \simeq 0.18 \mathrm{~s}$ ), so the transmitted wave can be isolated.

The time histories of the transmitted and reflected waves using the C/D nozzle are illustrated in figure 10: with a long termination figure $10(\mathrm{a})$, the upstream pressure $p_{u}$ shows the usual direct noise increasing with mass flow rate at times up to $0.1 \mathrm{~s}$, followed by the interference with the indirect noise appearing according to the convection time to the nozzle starting around 0.3 $\mathrm{s}$. The signal measured by the downstream transducer $p_{d}$ is observed in the inset to the figure. This acquired signal is therefore a combination of the transmitted direct and indirect noise. At

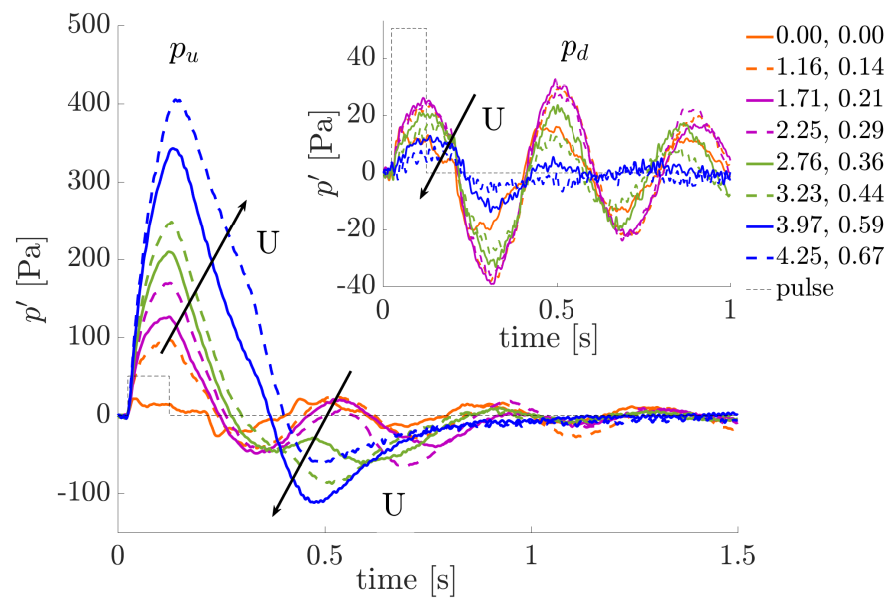

(a)

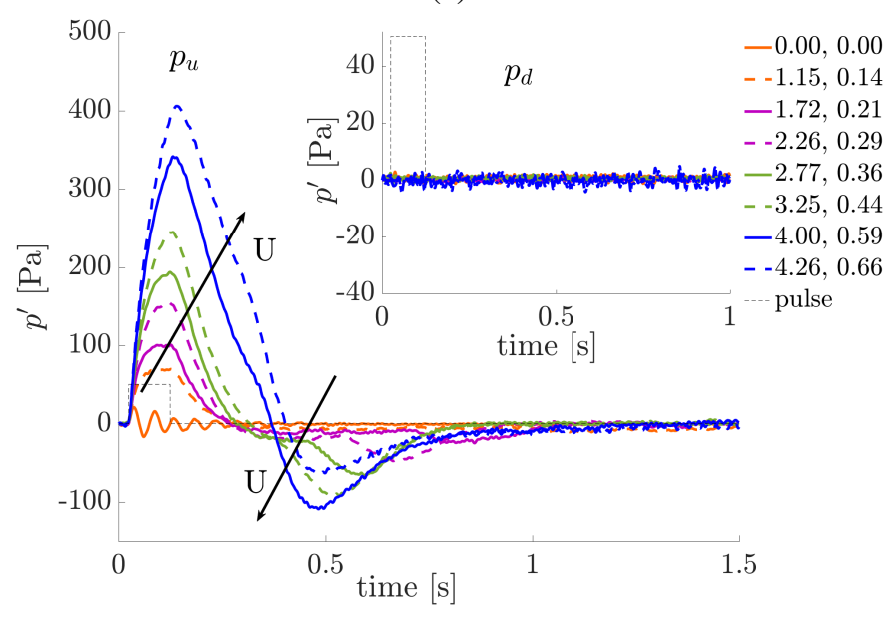

(b)

FIGURE 10. PRESSURE OSCILLATIONS ACQUIRED UPSTREAM $\left(p_{u}\right)$ AND DOWNSTREAM $\left(p_{d}\right)$ OF THE C/D NOZZLE. CONFIGURATION $\gamma$ WITH (a) AND WITHOUT (b) THE ANECHOIC TERMINATION RESPECTIVELY. INSET: DOWNSTREAM PRESSURE $\left(p_{d}\right)$. LEGEND: VELOCITY [M/S] AND THROAT MACH NUMBER [-]

times before the reflection from the open end of the long tube appears $\left(t<t_{a}\right)$, the signal can be interpreted as resulting from the original pulse. In contrast with the upstream pressure trace, the signal decreases with increasing flow rate, even though, as shown in figure 5, the direct and indirect noise travelling pulses should both increase. This would suggest that the acoustic transmissivity of the nozzle decreases with the flow, while its reflectivity (and the absorption) increases [25]. The measured values of reflected and transmitted waves are addressed further on. Figure 10(b) for the tube without the long extension shows the upstream 


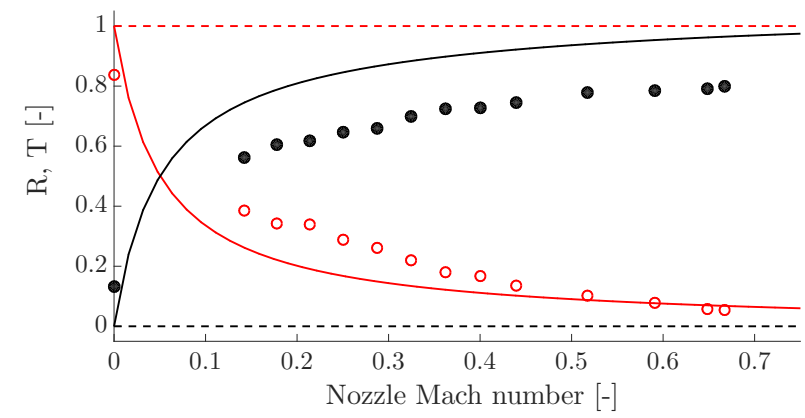

FIGURE 11. TRANSMISSIVITY $\left(T_{n}, \quad\right.$ RED LINES $)$ AND REFLECTIVITY $\left(R_{n}\right.$, BLACK LINES) OF THE CONVERGENT/DIVERGENT NOZZLE: COMPARISON BETWEEN EXPERIMENTAL DATA (SYMBOLS, CONFIGURATION $\gamma$ ) AND ANALYTICAL MODELS, ISENTROPIC ( [9], DASHED LINES) AND NON-ISENTROPIC ( [25], SOLID LINES)

pressure $p_{u}$ very similar to all other nozzles, but a zero measurement for the downstream pressure $p_{d}$ (inset). This is a result of the immediate reflection of the acoustic wave at the open boundary.

We can now turn back to figure 9 and compare the peak amplitudes of the upstream direct noise for the C/D nozzle cases with the convergent nozzle cases. For cases C/D N1 and N2, lower upstream pressures are detected in comparison with the convergent nozzles and orifices for low flow rates, rising to higher values increasing the flow rates. Therefore, for low flow rates the presence of the diffuser has a significant impact on the signal, while increasing flow rates the system behaves essentially as if there were no divergent section, even though the flow is always subsonic at the throat in the test conditions analysed. We now explain the results in terms of the measured transmissivity and reflectivity of the signal.

\subsubsection{Transmissivity and reflectivity of entropic} pulses The availability of pressure measurements upstream $(u)$ and downstream $(d)$ of the C/D nozzle allows the direct determination of the transmissivity and reflectivity of the low frequency acoustic perturbations through the nozzle. The transmission coefficient $\left(T_{n}\right)$ of a single pulse $p_{u}^{\prime}$ through a convergent/divergent nozzle is defined as

$$
T_{n}=\frac{p_{d}^{\prime} / \gamma \overline{p_{d}}}{p_{u}^{\prime} / \gamma \overline{p_{u}}}
$$

Since the experimental measurements may involve multiply reflected waves, it is necessary to deconvolute the measurements relatively to those of a single pulse. Rolland et al. [17] showed that, for a case where the acoustic time through the upstream tube is much smaller than the pulse interval, and where the upstream reflection coefficient $R_{u}$ is close to unity (as is the present case), the single pulse transmission coefficient $T_{n}$ can be related to ratio of the peak pressures measured upstream and downstream (from direct noise) via the following relation:

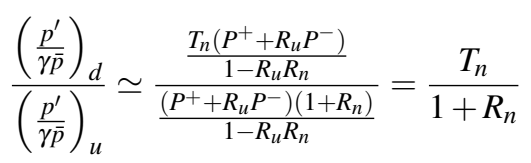

The reflection coefficient of the nozzle $R_{n}$ can separately be evaluated using:

$$
\left(\frac{p_{\text {max }}^{\prime}}{\gamma \bar{p}}\right)_{u} \simeq \frac{\left(P^{+}+R_{u} P^{-}\right)\left(1+R_{n}\right)}{1-R_{u} R_{n}}
$$

The reflection coefficient of the upstream end has been evaluated in a separate experiment as $R_{u}=0.97$. The values of $\left(P^{+}+R_{u} P^{-}\right)$are extracted from the measurements in figure 5(a) for each flow rate.

The resulting measured transmissivity and reflectivity of the $\mathrm{C} / \mathrm{D}$ nozzle are shown as symbols in figure 11 . The measurement results can be compared with two different models: (a) the limiting expressions offered by the isentropic, compact nozzle model of Marble and Candel [9] (dashed lines), and (b) the nonisentropic expression by Durrieu et al. [25] (solid lines). The equations for the reflection and transmission coefficients of a single wave in the isentropic, compact model of Marble and Candel are given as:

$$
\begin{aligned}
R_{n} & =\frac{M_{d}-M_{u}}{1-M_{u}} \frac{1+M_{u}}{M_{d}+M_{u}} \frac{1-0.5(\gamma-1) M_{u} M_{d}}{1+0.5(\gamma-1) M_{u} M_{d}} \\
T_{n} & =\frac{2 M_{d}}{1+M_{d}} \frac{1+M_{u}}{M_{d}+M_{u}} \frac{1+0.5(\gamma-1) M_{d}^{2}}{1+0.5(\gamma-1) M_{u} M_{d}}
\end{aligned}
$$

Where $M_{u}$ and $M_{d}$ are the upstream and downstream Mach number. Clearly, for a subsonic isentropic nozzle with the same upstream and downstream areas, as is the case in the present experiments, as $M_{u}=M_{d}$ no acoustic waves are propagated upstream of the nozzle $\left(R_{n}=0\right)$ and all acoustic energy is transmitted downstream $\left(T_{n}=1\right)$. The model by Durrieu et al. [25] instead takes into account the losses in total pressure occurring in the diverging section: the kinetic energy of the flow at the throat is not totally converted into total pressure downstream of the nozzle as the flow separates.

The non-isentropic model of Durrieu et al. is clearly in good agreement with the measurements. As the flow increases, the transmitted energy fraction becomes negligible, as it is dissipated by the effect of the jet, which does not follow the diverging profile. The differences between the experimental data and the Durrieu's model most likely arise because the latter is derived for an 
orifice plate, which creates more acoustic losses than the current nozzle. In contrast, the isentropic, compact model of Marble and Candel [9] cannot capture the behaviour of the partially reflecting/transmitting waves. Vorticity and recirculation may occur in the diverging section, and increase with flow rate, as suggested by the increase in the static pressure upstream of the nozzle (figure 7). Although the angle of the divergence is small $\left(\alpha \simeq 4.5^{\circ}\right)$, it is likely that the flow separates from the wall, causing additional turbulent noise and absorption of acoustic energy [31,32], in particular at low frequency [27]. Once the acoustic energy is converted into vortical energy, it is subsequently dissipated into heat, without further interaction with the acoustic field $[29,32]$. Bechert [31] concluded that if the diffuser is smaller than the acoustical wavelength, the device tends to behave as if no diffuser were there. Simulations of converging nozzles show that, if the section varies abruptly, areas similar to recirculation bubbles may appear, which absorb the incident sound waves, invalidating the quasi 1D hypothesis. These theoretical and experimental observations suggest that the theory of Marble and Candel should not be applied to convergent/divergent nozzles under similar conditions.

\subsubsection{Transmitted and reflected indirect noise} The compact isentropic nozzle theory [9] predicts that, for $M_{u}=M_{d}$, no indirect noise should be detected either upstream or downstream of the nozzle, provided that the entropy waves are not dissipated through the nozzle. This results from the fact that the acceleration and deceleration of the hot spots produce sound waves with the same amplitude and opposite sign, which cancel out under isentropic conditions. Yet the experimental data ( $p_{u}$ in figure 10) shows that indirect noise can still be clearly observed in the upstream traces. This means that there is no cancellation of upstream and downstream waves. Ideally, a direct observation of the decelerating entropy wave would confirm this observation. The picture for the downstream signal is somewhat less clear. Considering the perturbation shown in (figure 10(a)), one would expect anechoic conditions to prevail for the signal $p_{d}$ during the period $t<t_{a}=0.18 \mathrm{~s}$, so that in principle both direct and indirect noise transmitted should be observed. Indeed, the direct noise is transmitted as shown in 10(a). Yet the reflected direct noise wave comes back at $0.18 \mathrm{~s}$, which overlaps with the expected time a decelerating positive entropy wave would be observed, making the interpretation more difficult. Although these results are not conclusive regarding the transmitted indirect noise, the non-isentropic characteristics, combined with the lack of indirect noise cancellation upstream of the nozzle suggests that in the current experiment either the transmitted indirect noise is negligible or that its effects are considerably lower with respect to the transmitted direct noise, in contrast to the findings of similar previous experiments [40]. Further experiments are needed to characterise the behavior of hot spot deceleration (if any exists) downstream of the divergent nozzle.

\section{CONCLUSIONS}

This work analyses the behaviour of pressure waves arising from the activation of a heating grid, which produces two main thermoacoustic effects: the impulsive heating generates compression waves (direct noise) and hot spots that, when accelerated, produce sound (indirect noise). A new effective and inexpensive method to isolate the single travelling pulses is developed in the form of a semi-infinite tube separating the upstream and downstream boundary. This generates a time-window in which the downstream end is silent (anechoic) for the roundtrip time of an acoustic wave, allowing to analyse how various parameters, such as mass flow rate and duct pressure, influence the pressure trace. The following conclusions can be extracted from the resulting experiments.

(a) The upstream reflected direct noise is largely independent of the shape of the end terminations, which behave as compact sources regardless of the contraction shape, provided that they have the same effective throat area.

(b) The transmissivity of the direct noise through a converging/diverging nozzle agrees well with the non-isentropic model of Durrieu et al., [25]. The isentropic model of Marble and Candel [9] is inadequate for determining the reflected and transmitted wave.

(c) Although it is not possible to directly measure the transmitted indirect noise and the generated indirect noise through the nozzle in the present experiment, the results show that the assumption of isentropic behaviour is inadequate, and would result in an underprediction of the upstream propagating indirect noise.

In order to create the ability to detect the transmissivity and generation of single entropic pulses, future work will include the addition of long tubes will be connected both upstream and downstream of the rig, to isolate single travelling waves and clearly assess the total amount of energy reflected, transmitted and dissipated. This will enable further insight to compare the theoretical predictions and experimental data on the acousticentropic transfer functions of nozzles.

\section{ACKNOWLEDGMENT}

Francesca De Domenico is supported by the Honorary ViceChancellor's Award and a Qualcomm/ DTA Studentship (University of Cambridge). Erwan Rolland is supported by an EPSRC DTA studentship (University of Cambridge). Experiments were partly funded by EPSRC grant EP/K02924X/1. 
TABLE 1. NOZZLE DESCRIPTION

Case
$L=24 \mathrm{~mm}$
Parameter
N3

\section{Appendix A: Orifice shape}

Table 1 shows a schematic of the different end terminations used in the experiments. The different nozzles and the thick orifice plate have been $\mathrm{CNC}$ manufactured from a PVC rod. The thin orifice plate, designed following ISO 5167 [35], is made of stainless steel. N4 is the convergent part of a nozzle optimised [39] to generate the highest indirect noise possible ${ }^{1}$.

\footnotetext{
${ }^{1}$ Unpublished result, personal communication of the authors with Maxime Huet, Onera, and Alexis Giacque, LMFA EC Lyon.
}

\section{REFERENCES}

[1] Lord Rayleigh, J., 1894. The Theory of Sound. Macmillan.

[2] Chu, B. T., and Kovasznay, L. C. G., 1958. "Non-linear Interactions in a Viscous Heat-Conducting Compressible Gas”. Journal of Fluid Mechanics, 3, pp. 494-514.

[3] Magri, L., O’Brien, J., and Ihme, M., 2016. "Compositional inhomogeneities as a source of indirect combustion noise". Journal of Fluid Mechanics, 799, p. R4.

[4] Kings, N., and Bake, F., 2010. "Indirect Combustion Noise: Noise Generation by Accelerated Vorticity in a Nozzle Flow". International Journal of spray and combustion dynamics, 2, pp. 253-266.

[5] Dowling, A. P., and Mahmoudi, Y., 2015. "Combustion Noise". Proceedings of the Combustion Institute, 35(1), pp. 65-100.

[6] Goh, C. S., and Morgans, A. S., 2013. "The Influence of Entropy Waves on the Thermoacoustic Stability of a Model Combustor". Combustion Science and Technology(July 2015), p. 120816123400009.

[7] Hochgreb, S., Dennis, D., Ayranci, I., Bainbridge, W., and Cant, S., 2013. "Forced and Self-Excited Instabilities from Lean Premixed, Liquid-Fuelled Aeroengine Injectors at High Pressures and Temperatures". In Proceedings of the ASME Turbo Expo IGTI, June 3-7, 2013, San Antonio, Texas, USA, pp. GT2013-95311.

[8] Polifke, W., Paschereit, C. O., and Döbbeling, K., 2001. "Constructive and Destructive Interference of Acoustic and Entropy Waves in a Premixed Combustor with a Choked Exit”. Journal of Acoustics and Vibration, 6(3), pp. 135146.

[9] Marble, F., and Candel, S., 1977. "Acoustic disturbance from gas non-uniformities convected through a nozzle". Journal of Sound and Vibration, 55(2), pp. 225-243.

[10] Stow, S. R., Dowling, A. P., and Hynes, T. P., 2002. "Reflection of Circumferential Modes in a Choked Nozzle". Journal of Fluid Mechanics, 467, pp. 1-25.

[11] Durán, I., Moreau, S., and Poinsot, T., 2013. “Analytical and Numerical Study of Combustion Noise Through a Subsonic Nozzle”. AIAA Journal, 51(1), pp. 42-52.

[12] Bohn, M. S., 1976. "Noise produced by the interaction of acoustic waves and entropy waves with high speed nozzle flows". PhD thesis, California Insitute of Technology.

[13] Bake, F., Richter, C., Mühlbauer, B., Kings, N., Röhle, I., Thiele, F., and Noll, B., 2009. "The Entropy Wave Generator (EWG): A reference case on entropy noise". Journal of Sound and Vibration, 326(3-5), pp. 574-598.

[14] Gaetani, P., Persico, G., and Spinelli, A., 2015. "Entropy Wave Generator for Indirect Combustion Noise in a HighPressure Turbine". In Proceedings of the 11th European Conference on Turbomachinery Fluid Dynamics and Thermodynamics.

[15] Knobloch, K., Werner, T., and Bake, F., 2015. "Noise Gen-
Copyright (C) 2017 by ASME 
eration in Hot Nozzle Flow". In Proceedings of the ASME Turbo Expo 2015: Turbine Technical Conference and Exposition GT2015-43702, pp. 1-14.

[16] De Domenico, F., Rolland, E. O., and Hochgreb, S., 2017. "Detection of direct and indirect noise generated by synthetic hot spots in a duct". Journal of Sound and Vibration, pp. 1-17.

[17] Rolland, E., De Domenico, F., and Hochgreb, S., 2016. "Theory and application of reverberated direct and indirect noise". [Pre-print](Available from wwwg.eng.cam.ac.uk/reactingflows/_downloads/Rolland_JFM 2016.pdf).

[18] Franzoni, L. P., and Elliott, C. M., 1998. "An innovative design of a probe-tube attachment for a 2 -in . microphone". The Journal of the Acoustic Society of America, 104(5), pp. 2903-2910.

[19] Knobloch, K., Lahiri, C., Enghardt, L., Bake, F., and Peitsch, D., 2011. "Hot-Acoustic-Testrig- A Unique Facility for Thermoacousti Research". In Proceedings of ASME Turbo Expo 2011 GT2011.

[20] Laumonier, J., Jean, P., and Hardouin, L., 1997. “An Active Anechoic Termination for Low Frequencies with Mean Flow 1.”. pp. 25-34.

[21] Duran, I., and Moreau, S., 2013. "Solution of the Quasi One-Dimensional Linearized Euler Equations using Flow Invariants and the Magnus Expansion". Journal of Fluid Mechanics, 723, pp. 190-231.

[22] Trilling, L., 1955. "On Thermally Induced Sound Fields". The Journal of the Acoustic Society of America, 27(3), pp. 425-431.

[23] Brown, M. A., and Churchill, S. W., 1995. "Experimental Measurements of Pressure Waves Generated by Impulsive Heating of a Surface". Aiche Journal, 41(2), pp. 205-213.

[24] Hwang, I., and Kim, Y., 2006. "Measurement of ThermoAcoustic Waves Induced by Rapid Heating of Nickel Sheet in Open and Confined Spaces". International Journal of Heat and Mass Transfer, 49(3-4), pp. 575-581.

[25] Durrieu, P., Hofmans, G., Ajello, G., Boot, R., Aurégan, Y., Hirschberg, A., and Peters, M. C., 2001. "Quasisteady Aero-Acoustic Response of Orifices.”. The Journal of the Acoustical Society of America, 110(4), pp. 1859-1872.

[26] Holman, J., 2002. Heat Transfer, ninth ed. McGraw-Hill.

[27] Salikuddin, M., and Ahuja, K. K., 1983. "Acoustic power dissipation on radiation through duct terminations: Experiments". Journal of Sound and Vibration, 91(4), pp. 479502.

[28] Salikuddin, M., 1990. "Acoustic Behaviour of Orifice Plates and Perforated Plates with Reference to LowFrequency Sound Absorption". Journal of Sound and Vibration, 139, pp. 361-381.

[29] Leung, R. C. K. Ã., So, R. M. C., Wang, M. H., and Li, X. M., 2007. "In-duct orifice and its effect on sound ab- sorption”. pp. 990-1004.

[30] Cummings, A., and Eversman, W., 1983. "High Amplitude Acoustic Transmission Through Duct Terminations : Theory". Journal of Sound and Vibration, 91, pp. 503-518.

[31] Bechert, D., 1980. "Sound Absorption Caused by Vorticity Shedding, Demonstrated with a Jet Flow". Journal of Sound and Vibration, 70(79), pp. 389-405.

[32] Howe, S., 1979. Attenuation of Sound in a Low Mach Number Nozzle Flow. Tech. rep.

[33] Howe, M. S., 1979. "On the Theory of Unsteady High Reynolds Number Flow Through a Circular Aperture". Proceedings of the Royal Society of London. Series A, Mathematical and Physical Sciences, 366(1725), pp. 205223.

[34] Yang, D., and Morgans, A., 2016. “An Analytical Model for the Acoustic Imedance of Circular Holes of Finite Length". In Proceedings of the 23rd International Congress on Sound and Vibration. Anthens, Greece, no. 10-14 July 2016, pp. 1-8.

[35] Reader-Harris, M., 2015. “Orifice Design”. In Orifice Plates and Venturi Tubes. p. 393.

[36] Banks-Lee, P., and Peng, H., 1988. "Length error analysis for impedance tube measurements". The Journal of the Acoustic Society of America, 85(April 1989), pp. 17691772.

[37] Boden, H., 1986. "Influence of errors on the twomicrophone method for measuring acoustic properties in ducts". The Journal of the Acoustical Society of America, 79(2), p. 541.

[38] Bloy, A. W., 1979. "The pressure waves produced by the convection of temperature disturbances in high subsonic nozzle flows". Journal of Fluid Mechanics, 94(03), p. 465.

[39] Giauque, A., Huet, M., Clero, F., Ducruix, S., and Richecoeur, F., 2013. "Thermoacoustic Shape Optimization of a Subsonic Nozzle". Journal of Engineering for Gas Turbines and Power, 135(10), p. 102601.

[40] Bake, F., Kings, N., Fischer, a., and Röhle, I., 2009. "Experimental investigation of the entropy noise mechanism in aero-engines". International Journal of Aeroacoustics, 8(1), pp. 125-141. 